\section{Adapting to Climate Change}

To the Editor Dr Patz and colleagues ${ }^{1}$ raised concerns about the adverse health effects of climate change. They reviewed the evidence on the effects to date of the future risks from a changing climate and the opportunities for effective action to reduce risks. Although the authors urged public health preparedness (adaptation) to climate change, there could be limits to the effectiveness of adaptation.

The present global burden of disease from climate change is relatively small, and it is often assumed that human beings' capacity to adapt has no limits. Despite health being vulnerable to the vagaries of climate, humans have adjusted their behavioral patterns and technologies to adapt to a diverse range of climates. ${ }^{2}$ However, projected anthropogenic climate change will pose new challenges that may be beyond existing coping capacities.

Some extreme weather events have increased heatrelated illness and deaths. Changes in local temperature and rainfall have altered the distribution of certain disease vectors. Current public health practices, policies, and infrastructure may come under great pressure with the growing risk of maladaptation. ${ }^{3}$

Recent assessments have identified an increased risk of experiencing tipping points, and the corresponding effects have been projected to be potentially catastrophic. ${ }^{4}$ Although there is little quantification of the adaptation potential of public health associated with projected rates and magnitudes of climate change, susceptible communities are already facing limits in their capacity to adapt to existing climate variability.

For example, the health of populations on small islands and in resource-poor countries has been shown to be particularly vulnerable as a result of increasing exposure to severe weather conditions. ${ }^{5}$ The reason for concern is that as global warming accelerates, increasingly more communities will approach their adaptation limits.

The article by Patz et $\mathrm{al}^{1}$ demonstrates that responding to and communicating the related health risks involves making decisions in a changing climate with continuing uncertainty about the timing and severity of the effects. Health professionals will need to better understand the risks of doing nothing and the benefits to public health of undertaking appropriate and timely action.

\section{Cunrui Huang, MD, PhD \\ Roger Street, MS \\ Cordia Chu, PhD}

Author Affiliations: School of Public Health, Sun Yat-sen University, Guangzhou, China (Huang); Environmental Change Institute, University of Oxford, Oxford, England (Street); Centre for Environment and Population Health, Griffith University, Brisbane, Australia (Chu).

Corresponding Author: Cunrui Huang, MD, PhD, School of Public Health, Sun Yat-sen University, 74 Zhongshan Rd, Guangzhou 510080, China (huangcunrui@hotmail.com).

Conflict of Interest Disclosures: The authors have completed and submitted the ICMJE Form for Disclosure of Potential Conflicts of Interest and none were reported.

1. Patz JA, Frumkin $\mathrm{H}$, Holloway $\mathrm{T}$, Vimont DJ, Haines $A$. Climate change:

challenges and opportunities for global health. JAMA. 2014;312(15):1565-1580.
2. McMichael AJ. Insights from past millennia into climatic impacts on human health and survival. Proc Natl Acad Sci U S A. 2012;109(13):4730-4737.

3. Huang C, Vaneckova P, Wang X, Fitzgerald G, Guo Y, Tong S. Constraints and barriers to public health adaptation to climate change: a review of the literature. Am J Prev Med. 2011;40(2):183-190.

4. Lenton TM. Early warning of climate tipping points. Nat Clim Chang. 2011;1:201209.

5. Costello A, Abbas M, Allen A, et al. Managing the health effects of climate change: Lancet and University College London Institute for Global Health Commission. Lancet. 2009;373(9676):1693-1733.

In Reply We agree with Dr Huang and colleagues that there are limits to human beings' capacity to adapt to climate change, especially if preventive measures are not at the level of full society, multisector interventions. Climate change is neither a conventional single disease agent or toxic exposure nor an individual behavior that can be changed through medical technology or classic public health interventions. It represents a risk that can undermine societal stability and disrupt lifesupporting natural resources.

We agree that large uncertainties exist in the capacity to adapt in the face of climate conditions never experienced in the past. Some unforeseen problems for adaptation were glimpsed during the 2003 European heat wave. Extreme temperatures melted electrical cables. Nuclear power plants struggled to keep reactors cool. ${ }^{1}$ Many adaptation measures that rely on technology, such as air conditioning, may not be so dependable as the mercury climbs to unprecedented levels.

We also agree regarding the urgency of actions on climate change. We do not assume that adaptation to climate risks and mitigation of climate-altering greenhouse gas emissions should be a stepwise progression; our article concluded that curbing fossil fuel combustion must occur quickly and at substantial levels. For example, according to the Intergovernmental Panel on Climate Change, to avoid heating the earth more than $2^{\circ} \mathrm{C}$, by 2050 global greenhouse gas emissions would need to be $40 \%$ to $70 \%$ lower than 2010 levels and emission levels near zero by $2100 .^{2}$

Although such policy measures may at first seem unrealistic, our analysis provided quantitative examples of very large health co-benefits from mitigation of greenhouse gases across at least 3 sectors: energy, transportation, and food systems.

Cleaner energy strategies directed at short-lived climate pollutants could avoid about 1 million to 4 million deaths worldwide annually by 2050 by reducing fine particulate pollution. ${ }^{3}$ In the United States, monetized human health benefits associated with lower fine particulate air pollution exposures, particularly by reducing coal burning, could offset the cost of lowcarbon policies by up to 10 -fold. ${ }^{4}$ In other words, the value of health dividends could swamp the costs of striving for an energy efficient, low-carbon economy.

Upward trends in noncommunicable diseases throughout the world stem in part from sedentary lifestyles aided by transportation systems designed for automobile-dependent travel and from diets high in animal products and low in vegetables and fruit. So in addition to air quality improvements, there are more opportunities for public health through the adoption of alternative modes of transportation, especially 
those that promote active transport by foot or bicycle, alongside effective public transportation, and through the promotion of more healthful diets.

In summary, increasing trends in noncommunicable diseases in parallel to increasing rates of fossil fuel-based energy consumption that are disrupting the earth's climate present daunting risks to civilization. Yet the interdependence of these challenges affords a golden opportunity to solve both simultaneously. Action must not be delayed.

Jonathan A. Patz, MD, MPH

Howard Frumkin, MD, DrPH

Andrew Haines, MBBS, MD

Author Affiliations: Global Health Institute, University of Wisconsin, Madison (Patz); School of Public Health, University of Washington, Seattle (Frumkin); Department of Social and Environmental Health Research, London School of Hygiene and Tropical Medicine, London, England (Haines).

Corresponding Author: Jonathan A. Patz, MD, MPH, Global Health Institute, University of Wisconsin, 1710 University Ave, Madison, WI 53726 (patz@wisc .edu).

Conflict of Interest Disclosures: The authors have completed and submitted the ICMJE Form for Disclosure of Potential Conflicts of Interest and none were reported.

1. Keller C. Place matters: mortality, space, and urban form in the 2003 Paris heat wave disaster. Fr Hist Stud. 2013;36(2):299-330.

2. Intergovernmental Panel on Climate Change. Climate Change 2014 : Mitigation of Climate Change. New York, NY: Cambridge University Press; 2014.

3. Shindell D, Kuylenstierna JCI, Vignati E, et al. Simultaneously mitigating near-term climate change and improving human health and food security. Science. 2012;335(6065):183-189.

4. Thompson TM, Rausch S, Saari RK, Selin NE. A systems approach to evaluating the air quality co-benefits of US carbon policies. Nat Clim Chang. 2014;4:917-923.

\section{Goals of Displaying Health Care Prices to Physicians}

To the Editor The price of medical care is an essential component in the assessment of value. Drs Riggs and DeCamp ${ }^{1}$ discussed concerns about the display of price to physicians. The discussion is confounded when expanded to the goal of informing patients and ensuring fairness.

The key stakeholder in the assessment of the value of health care services is the patient. The physician's role as expert is to recommend the most cost-effective evaluation and management strategy. The authors' initial question of which price is right reflects that perspective.

The goals of price transparency for physicians and patients differ. The goal of price provision to the physician should be to inform his or her recommendation of the most costeffective tests or procedures. Some medical societies have provided appropriateness criteria for various tests that incorporate cost and outcomes. For this use, the indication of an actual dollar amount is unnecessary; the use of price indicators (eg, $\$, \$, \$ \$$ ) rather than actual prices can provide the aspect of price relevant to a physician's decision to recommend a particular test or procedure.

In discussion with a particular patient, however, the actual charges are pertinent. The authors suggested that if a patient is less well insured, the display of the price may decrease the chance of him or her receiving a test (compared with an insured patient) and therefore be unfair. This would only be unfair if the decision is made by the physician alone, rather than in consultation with the patient.

If the patient declines a recommended aspect of care based on the price, this may be unfair, but it is an inappropriate criticism of price transparency. Because individuals in the population have various levels of wealth and insurance coverage, the process of shared decision making can only maximize patient-centered value in the setting of their individual means and the bills they will confront.

Differential pricing may be unfair, but it reflects the underlying unfairness of the distribution of wealth and insurance coverage within the population; making prices transparent is not unfair.

\section{Eric K. Hoffer, MD}

Author Affiliation: Vascular and Interventional Radiology, DartmouthHitchcock Medical Center, Lebanon, New Hampshire.

Corresponding Author: Eric K. Hoffer, MD, Department of Radiology, Dartmouth-Hitchcock Medical Center, One Medical Center Drive, Lebanon, NH O3756 (hoffer@hitchcock.org).

Conflict of Interest Disclosures: The author has completed and submitted the ICMJE Form for Disclosure of Potential Conflicts of Interest and none were reported.

1. Riggs KR, DeCamp M. Providing price displays for physicians: which price is right? JAMA. 2014;312(16):1631-1632.

In Reply We agree with Dr Hoffer that prices are an essential component in assessing value and in general have no objection to the use of price displays. Rather, we argued in our Viewpoint that since different displayed "prices" (eg, hospital list price vs Medicare fee amount) might differentially affect physician behavior, it is important to consider how different prices might operate in practice and weigh the ethical implications of each.

We also agree that patients are arguably the most important stakeholder when assessing value and that physicians should engage with patients in discussing the out-of-pocket costs associated with their care. ${ }^{1}$ However, in some circumstances, robust shared decision making may not be possible. For example, patients in acute care hospitals or intensive care units may be unable or uninterested in weighing cost tradeoffs. In a recent survey of hospitalized patients, more than $70 \%$ indicated that they preferred to leave medical decision making to their physician. ${ }^{2}$

Uncertainty over the status of patients' deductibles, coinsurance, and eventual out-of-pocket responsibilities further complicates truly informed shared decision making. Recognizing that at least some price displays will operate mainly at the physician level, our intent was to bring attention to the ethical considerations involved, such as transparency, protecting patient well-being, and ensuring fairness.

Hoffer makes a novel suggestion that general indicators of price (eg, displaying \$ vs $\$$ \$ vs $\$ \$$ ) could suffice to inform value-based decision making. Particularly if small variations in price (such as displaying $\$ 30$ vs $\$ 40$ for a chest radiograph) do not affect physician behavior, we agree such strategies are simpler and worth exploring. However, when potential prices 